\title{
Design and Implementation of Power Harmonic Monitoring System based on Robust Kalman Filtering
}

\author{
Tingting Huang ${ }^{1}$, Ming Bai ${ }^{1}$,* Zhenxian Zhang ${ }^{1}$ and Yongqiang $\mathrm{Ye}^{1}$ \\ ${ }^{1}$ Guangzhou Maritime Institute, Guangzhou, Guangdong, 510725, China
}

\begin{abstract}
The inverters consisted of Power Electronic Devices are widely used in dredger, which makes Dredger Power System lots of Upper Harmonic. In order to achieve precise harmonic monitoring of cutter suction dredger power system, the paper proposes a power harmonic monitoring system based on Robust Kalman Filtering (RKF). In the simulation experiments, it showed that the Robust Kalman Filtering (RKF) is superior to the Kalman Filtering (KF) because the optimal estimate value is almost in agreement with the ideal value after adding the RKF. It gives experimental set-up and algorithm principle of RKF, and explains the correctness and feasibility of Power Harmonic Monitoring System based on Robust Kalman Filtering in the theory.
\end{abstract}

\section{Introduction}

As we all know, cutter suction dredger performs a major part in the field of dredging engineering in harbors, fairways, and land reclamation, especially in port construction and waterway reclamation projects $[1,2]$. Therefore, we can see easily dredger is the vital device to dredging engineering, and with the fast development of national economy, it is required to improve manufacturing technique of dredging engineering. The massive application of power electronic technology on ship has caused harmonic problems [3], and the problems have become more and more serious. Many marine accidents have happened in the world due to harmonic problems. Moreover, there are so many parameters in cutter suction dredger operation that it is difficult to guarantee the stability of production [4]. In order to achieve precise harmonic measuring and gain an optimal state estimation [5], the cutter suction dredger adopts a new algorithm principle named RKF, which is superior to conventional Kalman filtering [6].

In this paper, we firstly designed an experimental setup namely harmonic monitoring system, which is based on LabVIEW platform by PC [7]. Then, we provided formulas of RKF. The rest of this paper, we implemented RKF algorithm based on MATLAB simulation environment, the simulation results show the Robust Kalman Filtering (RKF) is superior to the Kalman Filtering (KF).

\section{Experimental Set-up}

In this section, the main interface of harmonic monitoring system for cutter suction dredger was drawn as shown in Fig.1.

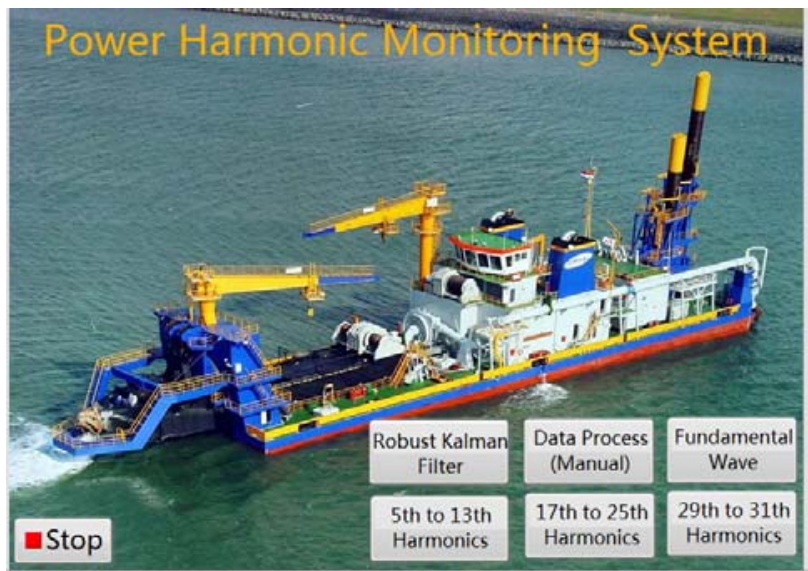

Fig1. main interface of Power Harmonic Monitoring System

\section{A dredger harmonic monitoring station}

The diagram of a dredger harmonic monitoring station was drawn as shown in Fig.2. Firstly, in order to measure dredger harmonic datas, Phasor Measurement Unit (PMU) based on the DSP data acquisition instrument connects with current and voltage transformer directly. Secondly, PMU communicates with power grid harmonic measuring system console through ModBus-RTU communication protocol and transfers the datas to PC. Lastly, LabVIEW and MATLAB to software programming write Human Machine Interface (HMI) programs, and the $\mathrm{KF}$ algorithm and its improved algorithm are built. The datas are displayed in real time, analyzed and stored. 


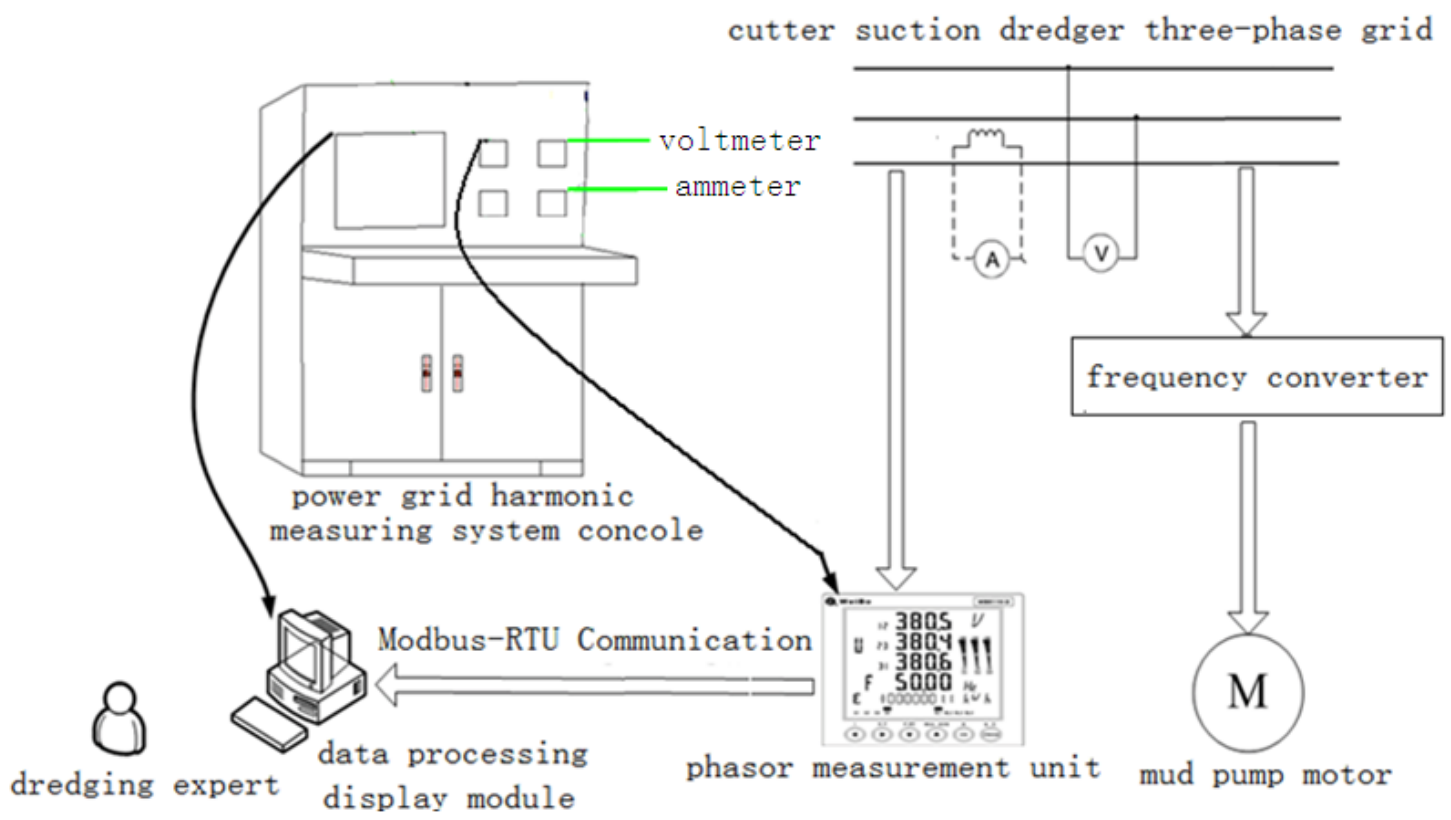

Fig2. Power Harmonic Monitoring System structure diagram.

\section{Algorithm Principle of RKF}

In order to verify the performance of the RKF, the simulation experiments were carried out on the MATLAB platform to construct the RKF. Fuzzy Inference System (FIS) has the ability to process fuzzy information based on the theory of fuzzy sets and fuzzy reasoning. RKF algorithm based on fuzzy adaptive reasoning was shown in Figure 3.

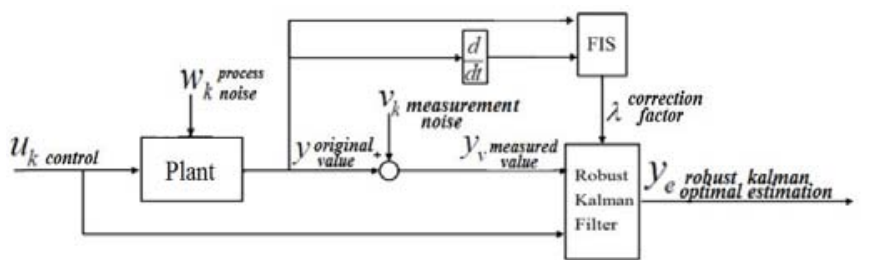

Fig 3. RKF algorithm based on fuzzy adaptive reasoning

Actually, process noise in the cutter suction dredger harmonic monitoring of ship power system is rarely a single Gaussian noise of average value of zero, because the dredged seabed contains a variety of soil mixed composition, including dug clay and sand digging, organic matter in soil and peat (silt) in dredging conditions. Besides, the state of the ocean varies from hour to hour in the course of dredging because of ocean currents, tides, weather and other factors, so generally, the noise is not Gaussian white noise of average value of zero. Strictly speaking, the ship power system of cutter suction dredger harmonic monitoring system embodies the sparse noise. The difference between the process noise including sparse noise and the measurement noise is that the measurement noise is caused by the component wear, sensor precision and line loss, etc. In order to better estimate the sparse noise, it is needed to design the RKF to solve this problem, and the RKF algorithm is proposed.
The principle of dredger RKF algorithm was shown in Figure 4.

Actually, Kalman filter is recursive, which means its prediction of the future relying on the state of the present (position, velocity, acceleration, etc) as well as a guess about what any controllable parts tried to do affect the situation (such as rudder or steering differential)[8]. Moreover, Kalman filter works by making a prediction of future , getting a measurement from reality ,comparing the two, moderating the difference, and adjusting its estimated value with the moderated value. The RKF algorithm model was here for exposition and reference as follows:

$$
\left\{\begin{array}{l}
P_{0}^{h}=\operatorname{Var}\left(x_{0}^{h}\right) \\
P_{k \mid k-1}^{h}=\left[A_{k-1}^{h} P_{k-1 \mid k-1}^{h}\left(A_{k-1}^{h}\right)^{T}\right]+Q^{h}{ }_{k-1} \\
G_{k}^{h}=P_{k \mid k-1}^{h} C_{k}^{h} /\left[C_{k}^{h} P_{k \mid k-1}^{h}\left(C_{k}^{h}\right)^{T}+R_{k}^{h}\right] \\
P_{k \mid k}^{h}=\left(I-G_{k}^{h} C_{k}^{h}\right) P_{k \mid k-1}^{h} \\
\hat{x}_{0}^{h}=E\left(x_{0}^{h}\right) \\
\hat{x}_{k \mid k-1}^{h}=A_{k-1}^{h}\left(\hat{x}_{k-1}^{h}\right)+B_{k-1}^{h}\left(u_{k-1}^{h}\right)+w_{k-1}^{h} \\
\tilde{X}_{k \mid k-1}^{h}=\lambda \hat{X}_{k \mid k-1}^{h}+(1-\lambda) \bar{X}_{k-1}^{h}, \lambda \in[0,1] \\
\hat{X}_{k \mid k}^{h}=\tilde{X}_{k \mid k-1}^{h}+G_{k-1}^{h}\left(z_{k}^{h}-D_{k}^{h} u_{k}^{h}-C_{k}^{h} \tilde{X}_{k \mid k-1}^{h}\right) \\
h=5,7, \ldots, 6 l \pm 1(l=1,2, \ldots), k=1,2, \ldots
\end{array}\right.
$$

Where $h$ means the time of harmonic, $p_{0}^{h}$ given is the state initial covariance, $p_{k \mid k-1}^{h}$ is the state prediction error covariance at time $\mathrm{k}$, and $P_{\mathrm{k} \mid \mathrm{k}}^{h}$ is the optimal state error covariance at time $\mathrm{k} . \mathrm{G}_{\mathrm{k}}^{\mathrm{h}}$ is the gain matrix at time k. $\hat{x}_{0}^{h}$ given is the state initial value. $\hat{x}_{k \mid k-1}^{h}$ is the state prediction by Kalman filtering at time $\mathrm{k}, \hat{x}_{k \mid k}^{h}$ is the state 


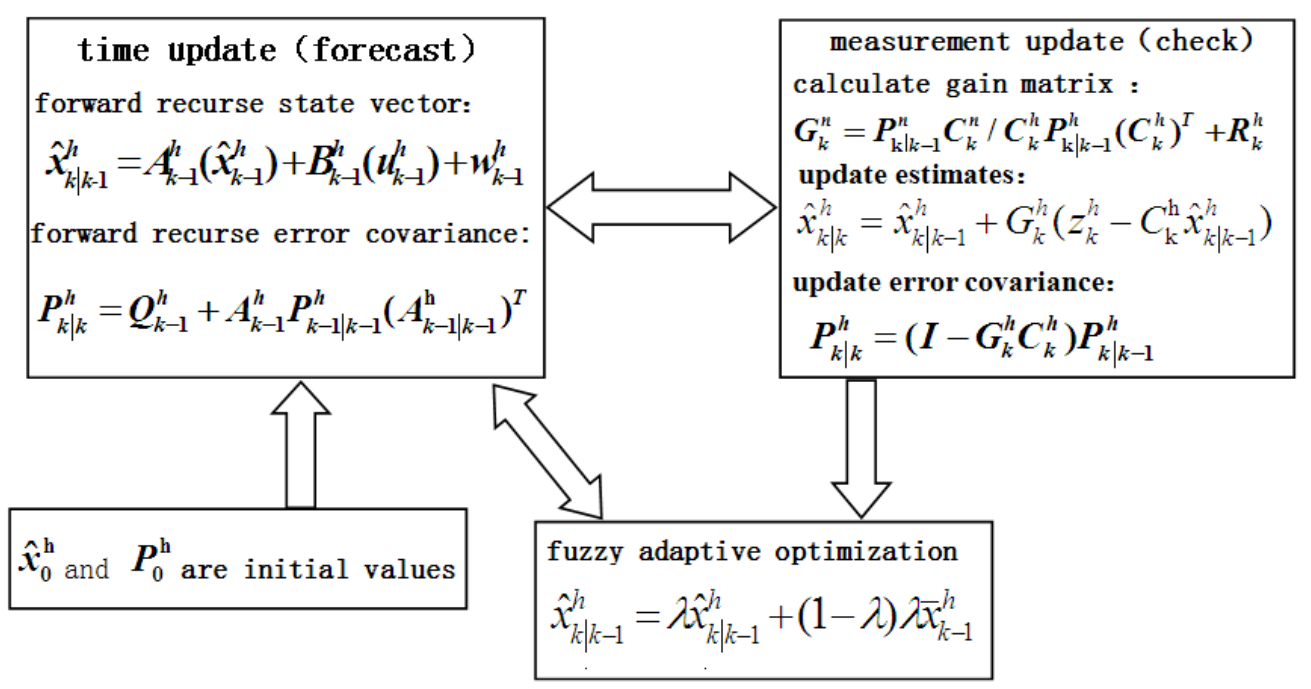

Fig 4. the principle of dredger RKF algorithm.

\section{Simulation Results and Discussion}

To better demonstrate the practicality of the design, and display the superiority of RKF. Lots of simulation experiments on cutter suction dredger were done. It is based on LabVIEW platform.

We regard the object as the transfer function $G(s)$ provided as follows:

$$
G(s)=\frac{15330}{s^{2}+180000 s}
$$

To imitate the fundamental wave of power system of cutter suction dredger, we inputted a sine signal with the amplitude of $975 \mathrm{~V}$. Different sparse noise will be added to the sine signal according to different working condition in which the cutter suction dredger works. What important is that we mainly adjust the correction factor to deal with the different sparse noise, and simulate by LabVIEW.
The experimental results based on RKF in fig.5 show that the fundamental wave optimal estimate value is more closer to the fundamental wave original value.

From the fig.6, it can be easily seen that the maximum of the Total Harmonic Distortion (THD) only reached $3.88 \%$, which is lower than $8 \%$ of China Classification Society (CCS) for requirement, and Root Mean Square (RMS) is near to $690 \mathrm{~V}$.

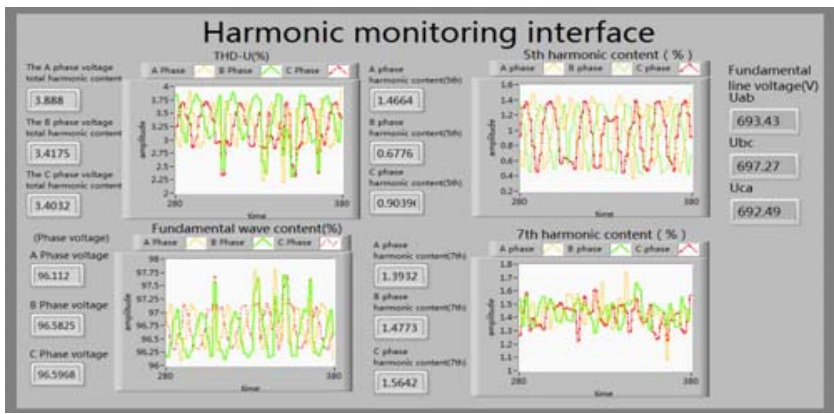

Fig 6. Interface of harmonic monitoring system

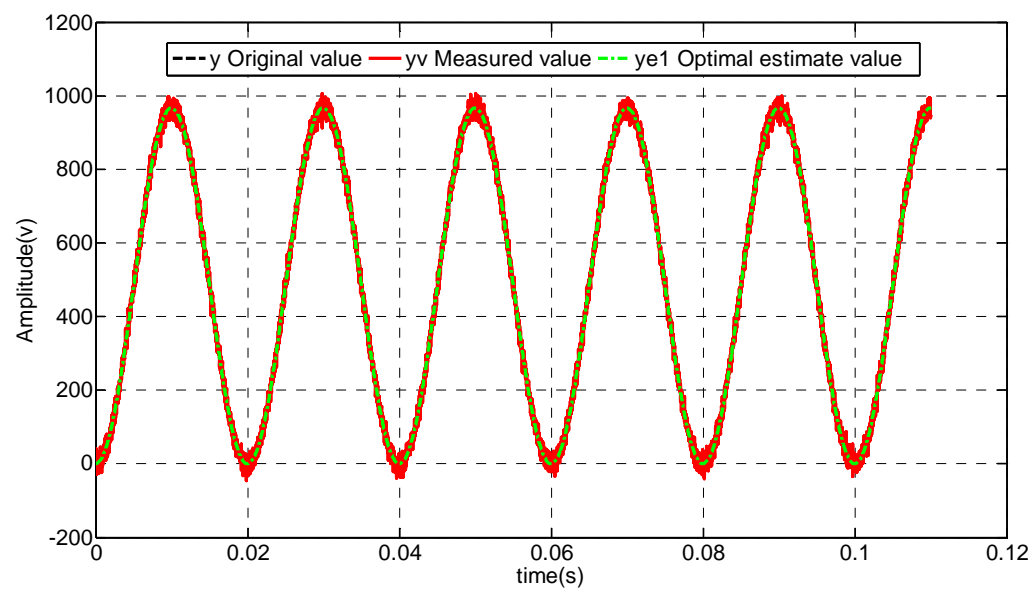

Fig 5. fundamental wave RKF effect diagram 
From the figure 7 , the optimal estimated value is almost in agreement with ideal value the after adding the RKF algorithm.

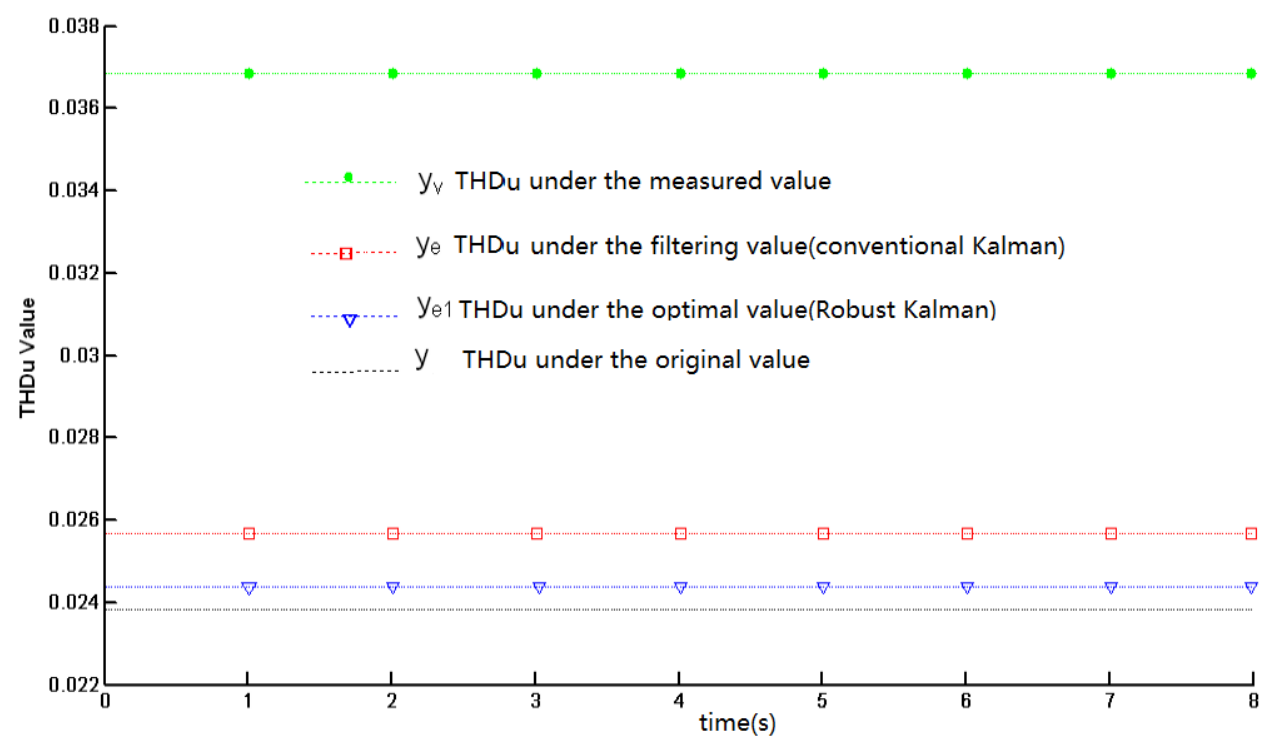

Fig7. numerical value comparison chart by RKF

In a word, the simulation results show that the fundamental wave and higher harmonic better approximate the ideal value by RKF. Thus, it's obvious that the RKF has better performances than the KF, and the optimal state estimation can be done.

\section{Conclusions}

After comparing the robust Kalman filter with the conventional Kalman filter and analyzing the above data seriously, there is no doubt that the cutter suction dredger harmonic monitoring system by RKF can work very precisely and the robustness of the system is greatly improved.

Based on Kalman filter of dredging ship power system harmonic monitoring system, the correctness and validity of the design by simulation can be verified. With high efficiency, high monitoring precision, low cost and wide application, the system has a wide range

of social and economic benefits in dredging ship power quality analysis as well as medium voltage high power inverter for the development of future dredging industry.

\section{Acknowledgements}

This work was financially supported by the Guangzhou Huangpu District Science and Technology Research Project (201237), which was supported by the construction project of strengthening universities with innovation in 2014 as well.

\section{References}

[1] Jianzhong Tang:Automatic monitoring and control of cutter suction dredger .Automation in Construction, 2008, Vol.18 (2), pp.194-203.

[2] Lei $\mathrm{Gu}$, Fu-sheng Ni,“Multimedia technology research in dredging simulation of cutter suction dredger”, Multimedia Technology (ICMT),2011 International Conference on, On page (s): 3139 3142.

[3] Yanqiong Song: Simulation of Harmonic Suppression for Power system of Marine Electric Propulsion.Basis of Theory and Applications on PID Control. Basic Automation[J],2007.

[4] Kaikai Li, Huanmin Xu,"Multivariate Principal Component Analysis for Production and Energy Consumption of Cutter Suction Dredger”,Applied Mechanics and Materials,Vols.644-650,pp.22112215, Sep. 2014.

[5] D.Simon, Optimal State Estimation - Kalman, H-infi nity, and Nonlin-ear Approaches. John Wiley and Sons, Hoboken, New Jersey, 2006.

[6] R. E. Kalman, “A new approach to linear filtering and prediction problems",Trans. ASME. Journal of Basic Engineering, vol. 82, no.1, pp. 35-45, 1960.

[7] Ming Bai,Jinhua Chen,“Design ADRC for Cutter Suction Dredger Cutter Motor Variable Frequency Drive System”,Advanced Materials Research,Vols. 1008-1009,pp.650-653,Aug.2014.

[8] Lindsay Kleeman: "Understanding and Applying Kalman Filtering”. Department of Electrical and Computer Systems Engineering Monash University, Clayton. 